\title{
Um estudo exploratório de ferramentas interativas como recurso didático na formação do estudante de medicina
}

\author{
An exploratory study of interactive tools as didactic resource in medical \\ student training
}

1 Paula Damato Dias Barroso pauladamato.b@gmail.com

1 Ítalo Bruno do Nascimento Moura

1 Cristina Ribeiro Dias Barroso

1 Rafael Teixeira dos Santos

1 Claudia Yamada Utagawa

1 Centro Universitário de Volta Redonda - UniFOA.

\section{Resumo}

As ferramentas interativas são recursos tecnológicos utilizados com a finalidade de ampliar o acesso ao conhecimento, podendo servir como alternativas complementares ao método tradicional de ensino. É nítido que, diante do desenvolvimento dos meios de comunicação e da evolução tecnológica, o compartilhamento de informações se torna cada vez mais simplificado. Nesse contexto, o presente trabalho tem o objetivo de apresentar as ferramentas interativas disponíveis na internet, de forma a expor sua aplicabilidade como recurso didático à formação acadêmica no curso de Medicina. Foi realizada uma busca na internet, entre abril e setembro de 2017, sobre as ferramentas interativas com conteúdo voltado para formação médica. As ferramentas encontradas foram caracterizadas por tipo de característica proposta: simulação, visualização e gamificação. Nove ferramentas foram avaliadas quanto à fonte/ fornecedor do produto, temática abordada, idioma utilizado, grau de intuitividade, grau de interatividade, disponibilidade para aplicativos de celulares e recursos para pessoas com deficiência visual.Constatou-se que as ferramentas interativas abordadas possuem grande potencial de difusão entre acadêmicos do curso de Medicina, visto que podem contribuir para a consolidação do conhecimento. No entanto, vale ressaltar que ainda são escassas as ferramentas de ensino em saúde com opções de utilização por pessoas com deficiência visual.

\section{Palavras-chave:}

Comportamento de utilização de ferramentas. Educação médica. Aprendizagem. Ensino.

\begin{abstract}
Interactive tools are technological resources used for the purpose of increasing access to knowledge, and can serve as complementary alternatives to the traditional teaching method. It is clear that, in the face of the development of the media and technological evolution, the sharing of information becomes more and more simplified. In this context, the present work has the objective of presenting the interactive tools available on the Internet, in order to expose its applicability as didactic resource to the academic training in the medical course. An internet search was conducted between April and September 2017 on interactive tools with content focused on medical education. The tools found were characterized by type of proposed feature: simulation, visualization and gamification. Nine tools were evaluated regarding the source / supplier of the product, thematic approach, language used, degree of intuitiveness, degree of interactivity, availability for mobile applications and resources for the visually impaired. It was found that the interactive tools discussed have great potential for dissemination among medical students, since they can contribute to the consolidation of knowledge. However, it is worth emphasizing that there are still few health education tools with options for use by people with visual impairment.
\end{abstract}

\section{Keywords:}

Tool use behavior. Medical education. Learning. Teaching.

\section{Como você deve citar?}

BARROSO, Paula Damato Dias et al.. Um estudo exploratório de ferramentas interativas como recurso didático na formação do estudante de medicina. Cadernos UniFOA, Volta Redonda, n. 39, p. 127-137, abril 2019. 
Um estudo exploratório de ferramentas interativas como

recurso didático na formação do estudante de medicina

\section{INTRODUÇÃO}

Na medicina, como em outras áreas do saber, observa-se um crescente acúmulo de conhecimento científico, associado à necessidade de desenvolvimento de competências e habilidades que demandam raciocínio crítico e reflexivo, sem perder de vista o comportamento ético e cidadão. Na área da saúde, reforça-se ainda a necessidade dealiar a teoriaà prática, numa visão integral do homem. (SOUZA, INGLESIAS, PAZIN-FILHO, 2014).

O uso de métodos inovadores na práxis pedagógica tem possibilitado maior engajamento dos alunos no processo ensino-aprendizagem, por meio da mobilização, da construção e das sínteses, contribuindo paraa vivência pessoal do aluno e para testar e retestar os conhecimentos aprendidos a partir do que já se conhece (AUSUBEL, 1967; SOUZA, IGLESIAS, PAZIN-FILHO, 2014).

Ainda nesse contexto, a relação professor-acadêmico é fundamental à aprendizagem no ambiente universitário, uma vez que estabelece o comprometimento com a construção do conhecimento, cabendo ao professor mediar e orientar o aluno, seja propondo desafios, seja incentivando atividades em grupo, por exemplo (BORGES, ALENCAR, 2014).

Em se tratando da formação do estudante de Medicina, a ampla gama de conteúdos abordados e o alto grau de integração e correlação que deve haver entre eles implica maior necessidade de diversificação dos métodos de ensino e de aprendizagem. 0 aluno deve tornar-se, assim, co-responsável pelo seu aprendizado e desenvolver autonomia e habilidades críticas e reflexivas para lidar com a resolução de problemas e a tomada de decisões (MOORE, 2002).

Assim, as ferramentas interativasabrangem websites (blogs, jogos, vídeo-aulas), aplicativos para computador e para dispositivos móveis, mídias sociais, entre outros. Elas consistem em meios de potencializar o aprendizado e dinamizar a maneira como o conhecimento é construído e veiculado, tanto nas salas de aula quanto no estudo individual.

Atualmente, isso é possível graças ao grande contingente de informações, que cresce exponencialmente em nível mundial, e à rápida evolução dos meios de comunicação e das áreas da eletrônica e da computação, o que possibilita a troca de informações à distância e de forma fácil e acessível. A ascensão dos recursos interativos demonstra a possibilidade de seu uso como ferramenta alternativa educacional promissoranas universidades, à medida que pode complementar - às vezes, até substituir - aulas tradicionalmente expositivas e fragmentadas, em que há grande dependência intelectual do aluno pelo professor (TORI, 2010).

O objetivo deste trabalho foi explorar a utilização de ferramentas interativas inovadoras disponíveis na internet, buscando analisar e categorizar a aplicabilidade de tais metodologias como recursos didáticos à aprendizagem e aperfeiçoamento da Educação Médica na formação do discente do curso de Medicina.

O presente estudo avaliou as diferentes ferramentas inovadoras, considerando a importância dos seus recursos didáticos para a formação crítica do estudante de medicina, servindo como base de orientação para o desenvolvimento de novas e melhores ferramentas e metodologias de ensino, além de auxiliar os alunos na escolha e na utilização de tais recursos. 


\section{METODOLOGIA}

Foi realizada, inicialmente, uma revisão bibliográfica através de pesquisas nos bancos de dados das bases indexadoras PubMed/MEDLINE eSciELO acercada utilização de ferramentas interativas como recurso didático na formação de alunos do curso de Medicina.Foram utilizadas as seguintes expressões de pesquisa: "simulação médica"; "gamificação"; "educação médica"; "tecnologias inovadoras"; "tecnologias interativas", entre abril e setembro de 2017. As pesquisas também foram realizadas em língua inglesa, sendo utilizadas as mesmas expressões de pesquisa.

Após essa etapa, foram realizadas buscas através da plataforma digital Google (https://www. google.com.br/) com as seguintes expressões de pequisa: "educação médica"; "jogos"; "simulação médica"; "atlas"; "pacientes virtuais"; "ferramentas interativas em medicina"; "anatomia". As pesquisas também foram realizadas em língua inglesa com as mesmas expressões de pesquisa: "medical education"; "games"; "virtual patients"; "medical simulation";"atlas";"interactive tools for medical students"; "anatomy".

Foram elencadas as principais ferramentas disponíveis na internet, sendo que os critérios absolutos de inclusão foram: disponibilidade gratuita na internet e desenvolvidas por instituições confiáveis. Em seguida, cada uma foi analisada quanto à fonte/fornecedor do produto, temática abordada (no campo médico), idiomas disponíveis, grau de intuitividade (facilidade de uso), grau de interatividade (recursos de áudio, vídeos ou imagens), disponibilidade para aplicativos de celulares e recursos para pessoas com deficiência visual (áudios de leitura).

Posteriormente, as ferramentas foram categorizadas em três tipos: ferramentas de simulação, de visualização e de gamificação. Essa classificação foi realizada após extensa leitura de artigos científicos, dentre eles, os de Sabbatini (1994), Dos Santos (2005), Flato (2011), Alves (2014) ePeres (2015).

Embora trabalhos que classificassem e abordassem os três tipos de ferramentas não tenham sido encontrados, o presente artigo considerouas principais técnicas disponíveis em ambientes virtuais de aprendizagem (AVA). Ainda que algumas dessas ferramentas possuam características que as classifiquem em mais de uma categoria, foi considerado o objetivo principal de seus recursos.

\section{RESULTADOS}

Foram selecionadas nove ferramentas de interesse para educação médica, respeitando-se os critérios de inclusão anteriormente mencionados.

Além disso, cada ferramenta foi testada e avaliada quanto as suas características individuais. Sendo assim, os temas abordados foram: quatro sobre casos clínicos diversos, uma sobre radiologia, três sobre anatomia humana e uma sobre cirurgia geral. 0 idioma predominantemente utilizado foi o inglês, sendo algumas ferramentas encontradas em língua portuguesa e em outras línguas (sendo possível a modificação no site, conforme preferência do usuário).

Quanto aos graus de intuitividade e interatividade, todas as ferramentas possuem fácil manejo de seus recursos, além de algumas disponibilizarem também recursos de áudio, imagem e vídeo. A todas elas foi atribuído médio ou alto grau de intuitividade e interatividade. 
Um estudo exploratório de ferramentas interativas como recurso didático na formação do estudante de medicina

Quanto à disponibilidade para aplicativos de celulares, quatro ferramentas possuem esse recurso, que é de grande valia, uma vez que aumenta a praticidade de utilização do material didático e o torna também facilmente portátil.

Finalmente, em relação aos recursos para pessoas com deficiências visuais, apenas uma das nove ferramentas selecionadas fornece, limitadamente, um recurso de áudio para a leitura do material apresentado.

Segue abaixo uma tabela comparativa e resumida das ferramentas selecionadas.

Tabela 1 - Relação comparativa das ferramentas e suas características.

\begin{tabular}{|c|c|c|c|c|c|c|}
\hline Tipo & Acesso (Link) & Desenvolvedor & Temática & $\begin{array}{l}\text { Aplicativo } \\
\text { para } \\
\text { celular }\end{array}$ & $\begin{array}{l}\text { Acessibilidade } \\
\text { para } \\
\text { pessoas com } \\
\text { deficiência } \\
\text { visual }\end{array}$ & Idioma \\
\hline Paciente Virtual & $\begin{array}{l}\frac{\text { http://www.nejm.org/ }}{\text { multimedia/ }} \\
\frac{\text { interactive-medical- }}{\text { case }}\end{array}$ & $\begin{array}{l}\text { The New } \\
\text { EnglandJournalof } \\
\text { Medicine }\end{array}$ & $\begin{array}{l}\text { Caso } \\
\text { Clínico }\end{array}$ & Não & Não & Inglês \\
\hline Paciente Virtual & $\begin{array}{l}\text { http://www.nejm.org/ } \\
\text { multimedia/interactive- } \\
\text { medical-case }\end{array}$ & $\begin{array}{l}\text { MedBiquitous } \\
\text { Europe, nove } \\
\text { universidades e a } \\
\text { Comissão Européia }\end{array}$ & $\begin{array}{l}\text { Caso } \\
\text { Clínico }\end{array}$ & Não & Sim (áudio) & Diversos \\
\hline Paciente Virtual & $\begin{array}{l}\frac{\text { http://vpsim.pitt.edu/ }}{\text { shell/ }} \\
\frac{\text { CaseList_Assignments. }}{\underline{\text { aspx }}}\end{array}$ & $\begin{array}{l}\text { Universityof } \\
\text { Pittsburgh }\end{array}$ & $\begin{array}{l}\text { Caso } \\
\text { Clínico }\end{array}$ & Não & Não & Inglês \\
\hline Radiopaedia & https://radiopaedia.org & Investling & & Sim & Não & Inglês \\
\hline BioDigitalHuman & $\begin{array}{c}\text { https://www.biodigital. } \\
\text { com }\end{array}$ & $\begin{array}{c}\text { Harvard Medical } \\
\text { School e empresas } \\
\text { Novartis, } \\
\text { Medtronic, } \\
\text { VeryWell, Boston } \\
\text { Scientific e Clinical } \\
\text { Works }\end{array}$ & $\begin{array}{l}\text { Anatomia } \\
\text { Humana }\end{array}$ & Sim & Não & Inglês \\
\hline InnerBody & $\begin{array}{l}\text { http://www.innerbody. } \\
\text { com }\end{array}$ & HowToMedia Inc. & $\begin{array}{l}\text { Anatomia } \\
\text { Humana }\end{array}$ & Não & Não & Inglês \\
\hline TouchSurgery & $\begin{array}{l}\text { https://www. } \\
\text { touchsurgery.com }\end{array}$ & TouchSurgeryLabs & $\begin{array}{c}\text { Cirurgia } \\
\text { Geral }\end{array}$ & Sim & Não & Diversos \\
\hline InsuOnline & $\begin{array}{l}\text { https://oge.mobi/ } \\
\text { insuonline }\end{array}$ & $\begin{array}{l}\text { Universidade } \\
\text { Estadual de } \\
\text { Londrina }\end{array}$ & $\begin{array}{l}\text { Caso } \\
\text { Clínico }\end{array}$ & Sim & Não & Português \\
\hline Anatogames & $\begin{array}{l}\text { http://anatogames. } \\
\text { blogspot.com }\end{array}$ & $\begin{array}{l}\text { Laboratório de } \\
\text { Anatomia de } \\
\text { Sorocaba }\end{array}$ & $\begin{array}{l}\text { Anatomia } \\
\text { Humana }\end{array}$ & Não & Não & Diversos \\
\hline
\end{tabular}

Fonte: autores, 2019. 


\section{DISCUSSÃO}

\subsection{Ferramentas de simulação}

A simulação médica utiliza modelos artificiais com o intuito de substituir o encontro com pacientes reais, através de atores ou da realidade virtual. A replicação de cenários de cuidados ao paciente em um ambiente próximo da realidade tem o objetivo de analisar as ações desempenhadas de forma segura e controlada (GABA, 2009). Os Pacientes Virtuais (PV) são ferramentas interativas desenvolvidas em computador que simulam situações clínicas, sendo encontrados em diversos sítios eletrônicos (ASSOCIATION OF AMERICAN MEDICAL COLLEGES, 2007). Os PV possuem grande potencial para ser uma forma inovadora e eficaz de ensino, auxiliando na formação dos alunos a partir de abordagens variadas de metodologia ativa (BARROSO, 2018). Abaixo estão detalhadas as três ferramentas selecionadas que abordam a temática da discussão de casos clínicos a partir de pacientes virtuais, sendo elas: Interactive Medical Cases, eViP e vpSim.

\subsubsection{Interactive Medical Cases}

A revista da Sociedade Médica de Massachusetts - The New EnglandJournalof Medicine - possui, desde 2009, um espaço em seu website apenas para seus casos médicos interativos, que funcionam basicamente como um PV. Encontrar essa ferramenta em seu website torna-se fácil, quando já temos conhecimento de sua existência. A plataforma "Interactive Medical Cases" exibe, aproximadamente, 50 casos clínicos interativos, sendo todos disponibilizados em língua inglesa.Possuem ilustrações individuais de cada paciente e indicam os pesquisadores responsáveis por seu desenvolvimento. Inicialmente, há a apresentação do caso, seguida da história clínica e do exame físico e seus achados. Em seguida, temos perguntas sobre o caso (qual conduta tomar, qual medicamento ou exame solicitar) e seu padrão se mostra linear, uma vez que, ao respondermos a pergunta, o sistema já nos mostra se está certa ou não e explica o porquê de cada uma delas, passando para o seguinte passo, sendo uma ferramenta intuitiva, de fácil utilização (INTERACTIVE MEDICAL CASES, 2017).

No decorrer do caso, são disponibilizados diversos exames para visualização como exames de imagem (tomografia computadorizada, radiografia), exames de sangue e histopatológicos, permitindo interatividade com seus usuários. Além disso, abordam também a questão do tratamento e seguimento do caso. No final, nos disponibilizam os "teaching points", que são pontos importantes aprendidos com o caso, além de fornecerem a pontuação com base em nossas respostas, comparando-as com a pontuação total de todas as pessoas que já realizaram o mesmo caso. Possui aplicativo correspondente para iOS. Não possui recursos para deficientes visuais (INTERACTIVE MEDICAL CASES, 2017).

\subsection{2 eViP}

O programa Electronic Virtual Patients (eViP) é uma colaboração entre nove universidades e a MedBiquitous, além de ter como co-fundador a Comissão Europeia. Cada caso possui um título, suas palavras-chave, o idioma usado e a instituição responsável por seu desenvolvimento. No link disponibilizado por cada caso clínico, é possível entrar na página e realizá-lo. A plataforma é bastante intuitiva, sendo de fácil utilização. Diferentemente do que foi dito sobre os "casos médicos interativos", a página eViP não possui uma homogeneidade entre seus casos, visto que são desenvolvidos por instituições distintas. Possui, aproximadamente, 400 pacientes virtuais em sua plataforma, sendo sua maioria em língua inglesa, além de sueco, romeno, alemão e polonês (EVIP, 2017).

Assim sendo, alguns apresentam realização linear, enquanto outros são não lineares; alguns possuem maior grau de interatividade, com vídeos, fotos e áudios, e outros não. 0 interessante é que, 
Um estudo exploratório de ferramentas interativas como

recurso didático na formação do estudante de medicina

ao final de todos, é possível visualizar opathway, que é a ordem de todas as etapas realizadas, que mostra como foi o desenvolvimento do caso, com maior ou menor dificuldade. Não possui aplicativo correspondente para celulares. Alguns casos possuem recursos para deficientes visuais, uma vez que dispõem de áudios para a leitura do texto em questão (EVIP, 2017).

\subsection{3 vpSim}

A plataforma vpSim, da Universidade de Pittsburgh, foi criada no ano de 2009 e atualmente possui 30 casos clínicos. Cada caso possui um título, o nome de seu criador e a data de quando foi enviado à plataforma. Quando selecionado, o caso abre uma página individual que dá acesso ao seu início. Todos os casos são em língua inglesa. Assim como a plataforma do eViP, o website possui casos bastante diferentes, alguns possuem áudio, vídeo e imagens, podem possuir mais ou menos perguntas e até mesmo uma pequena pesquisa de satisfação ao final da simulação. A intuitividade da plataforma é boa, visto que é de fácil manuseio das ferramentas disponíveis (VPSIM, 2017).

São em sua totalidade de caráter linear, seguindo apenas uma linha fixa de desenvolvimento, em que as respostas incorretas são corrigidas e o caso é passado para a próxima etapa (BARROSO, 2018). Dessa forma, possui interatividade moderada, uma vez que não é possível modificar o curso dos casos clínicos. A plataforma não possui aplicativo correspondente para celulares e não possui recursos para deficientes visuais (VPSIM, 2017).

Os modelos de simuladores podem ser classificados como simuladores de baixa ou alta tecnologia. $\mathrm{O}$ uso de atores com roteiros pré-definidos, recursos auditivos ou visuais compõem os de baixa tecnologia. Já os de alta tecnologia, consistem no uso de manequins e programas de computadores capazes de reproduzir fielmente situações médicas (FLATO, 2011), como é o caso dos programas anteriormente citados.

A fragmentação do conhecimento em especialidades e o aprendizado médico baseado em técnicas passivas, como aulas teóricas e testes escritos, comprovadamente diminuem a retenção do conhecimento e sua aplicabilidade na prática (KHAN, 2011). Através das simulações, é possível simplificar o aprendizado de alunos e profissionais de saúde, além de minimizar complicações decorrentes de intervenções ou tratamentos, aumentando a segurança aos pacientes (AGGARWAL, 2010).

Assim, o uso de simulações que se aproximem da vida real proporciona melhor preparo dos profissionais frente a situações de agravo que exijam atuação rápida e eficaz. A redução de incidentes ocorre através de medidas de prevenção de erros de fatores humanos, da detecção precoce dos problemas, da minimização de lesões e da melhora no conhecimento (DOURADO, 2014). As ferramentas apresentadas corroboram com tais informações, uma vez que possibilitam que seus usuários aprendam com os erros e que solucionem as dúvidas. As simulações aproximam os alunos e profissionais das situações práticas reais com pacientes, proporcionando maior consolidação e aplicação do aprendizado.

\subsection{Ferramentas de visualização}

São programas facilmente disponíveis através de dispositivos com acesso à internet. As ferramentas oferecem um maior número de recursos visuais e interativos ao usuário, o que as torna cada vez mais difundidas entre estudantes e profissionais. Um dos mais conhecidos exemplos são os atlas digitais com visualização das estruturas em duas ou em três dimensões, alguns contendo até animações de funções orgânicas (MONTEIRO, 2006). A seguir, foram abordadas as três ferramentas de visualização selecionadas: Radiopaedia, sobre radiologia e BioDigitalHuman e InnerBody, sobre anatomia humana. 


\subsubsection{Radiopaedia}

O site foi lançado em dezembro de 2005 e atua disponibilizando numerosos artigos e casos clínicos de temática radiológica em língua inglesa. Foi fundado pelo neuroradiologista australiano, também professor da Universidade de Melbourne, Frank Gaillard, e a empresa que detém o site é a Investling. Cada caso clínico apresenta uma ou mais imagens radiológicas para auxílio diagnóstico, incluindo radiografias, ultrassonografias, tomografias computadorizadas e ressonâncias magnéticas; em alguns casos, há ilustrações. 0 acesso aos casos não requer nenhum tipo de cadastro. Contudo, é permitido que quaisquer visitantes contribuam com casos que conheçam e, para isso, é preciso se cadastrar. Antes de ser postado aos visitantes, cada caso é devidamente analisado e precisa ser autorizado pelos editores da página, pessoas com formação acadêmica na área médica. Antes de escolher um caso, o visitante pode ler o nome da pessoa que o enviou, os tipos de imagem radiológica que ele encontrará e o grau de certeza diagnóstica. Tem alto grau de intuitividade e a interatividade varia de acordo com a imagem disponível: quando se trata de tomografias e ressonâncias, é possível visualizar diversos cortes, de forma semelhante à que o radiologista os manuseia durante a realização dos exames; o download das imagens está disponível e facilita o estudo estático, como é o caso das radiografias, pois se torna possível ampliá-las. Possui aplicativo somente para iOS e não dispõe de recursos para deficientes visuais (RADIOPAEDIA, 2017).

\subsubsection{BioDigitalHuman}

A empresa BioDigital Inc. foi fundada em 2002, por Frank Sculli e John Qualter, mas a criação da plataforma BioDigitalHuman só viria em 2011 e tem colecionado prêmios nos campos da saúde, educação e pesquisa desde 2012. Trata-se de um software interativo 3D que conta com a colaboração da Harvard Medical School e das empresas Novartis, Medtronic, VeryWell, Boston Scientific e eClinical Works. Está disponível somente em língua inglesa. Essa ferramenta tem alto grau de intuitividade, além de contar com um breve tutorial de uso para os iniciantes. Oferece a visualização de toda a anatomia humana, em três dimensões, e permite ampliar, girar e selecionar somente as partes do corpo que serão estudadas. Toda a anatomia normal e parte da patológica são gratuitas, mas a página oferece também setores com animações pagas de anatomia patológica e de fisiologia. Não requer cadastro, mas vale mencionar que o software é considerado pesado para os dispositivos de acesso - computadores, tablets e telefones celulares -, o que lentifica seu uso e afeta a qualidade da interatividade, que, do contrário, seria sempre muito eficiente. Possui aplicativo para Android e iOS. Não possui recursos para portadores de deficiências visuais (BIODIGITALHUMAN, 2017).

\subsubsection{InnerBody}

É uma plataforma online criada em 1997, pertencente à empresa HowToMedia Inc., com sede no estado da Califórnia (EUA). É uma ferramenta de língua inglesa, inteiramente gratuita, abrange os 13 sistemas anatômicos do organismo e foi diversas vezes premiada. As animações 3D trazem também informações teóricas para leitura e permitem ampliação, rotação e seleção de determinadas partes do corpo, separadamente. É, portanto, bastante intuitiva e a interatividade é muito satisfatória, ainda que, a partir de certo grau de aumento da imagem, não é mais possível fazer a rotação adequada da parte visualizada. 0 programa é mais rápido que o anteriormente apresentado, exceto quando se trata da rotação da estrutura: nesse quesito, sua interatividade torna-se equivalente ao anterior. A página não requer cadastro, está disponível somente na internet e não dispõe de recursos para deficientes visuais (INNERBODY, 2017).

Segundo Dos Santos Nunes (2011), o desenvolvimento tecnológico tem alterado diversas práticas na área de saúde (educação, treinamento, diagnóstico). Nesse sentido, as ferramentas apresentadas 
agregam novas possibilidades ao estudo e à construção do conhecimento, à medida que proporcionam a visualização e a interação com modelos virtuais de anatomia humana, tornando mais claro e realista o conteúdo escrito estudado, e ampliam o contato com exames radiológicos e seus diagnósticos, como forma de treinamento para a futura prática médica.

\subsection{Ferramentas de gamificação}

As ferramentas de gamificação no âmbito da medicina consistem na aplicação da tecnologia intrínseca dos games para ampliar a interação dos usuários em um ambiente pedagógico e educacional. Assim, os eletrônicos já disseminados atualmente, como celular, tablet e computador, oferecem informações e recursos ao ensino com ferramentas ativas ao criar um ambiente estimulante e produtivo (KAPP, 2012). Adiante, serão abordadas três ferramentas de gamificação: TouchSurgery (cirurgia geral), InsuOnline (clínica médica) e Anatogames (anatomia humana).

\subsubsection{TouchSurgery}

A plataforma TouchSurgery é um simulador cirúrgico móvel interativo, desenvolvido pela KINOSIS, que fornece um guia realista e detalhado para cada etapa de diversos procedimento na área de cirurgia, utilizando arte gráfica em três dimensões de grande resolução, realizando a associação entre anatomia e procedimentos cirúrgicos e foi desenvolvido para profissionais da área da saúde. Os usuários podem aprender rapidamente diversos processos cirúrgicos, através de tutoriais demonstrativos e, em seguida, testar seus conhecimentos recém-adquiridos, repetindo o passo-a-passo assistido. Os tutoriais também trazem a descrição de cada etapa da cirurgia apresentada. Está disponível nas línguas inglesa, espanhola, portuguesa, ucraniana, polonesa, chinesa e russa. Além de permitir avaliar o conhecimento através do modo "Teste", posteriormente, através de um ranking, o usuário consegue comparar seus resultados. TouchSurgery tem sido usado em todo o mundo, fornecendo treinamento personalizado para programas de residência (programas de treinamento cirúrgico hospitalar). Vale destacar que o aplicativo também é gratuito em iOs e Android. Não possui recursos para deficientes visuais (TOUCHSURGERY, 2017).

\subsubsection{InsuOnline}

O InsuOnline é um jogo com o objetivo de melhorar o conhecimento acerca do uso da insulina. É eficaz no ensino da prescrição e manejo do paciente diabético em uso de insulina e dispõe de diversos casos clínicos, facilitando a aprendizagem do usuário. 0 jogo está disponível apenas em língua portuguesa e baseia-se em diretrizes sobre diabetes adaptadas à realidade da Atenção Primária brasileira. A ferramenta foi desenvolvida com base nos princípios da Educação de Adultos, para garantir a máxima eficiência educacional, potencializando a motivação, o engajamento e o aprendizado dos jogadores. Sua eficiência educacional foi testada por meio de uma pesquisa, cujos resultados mostraram o aprendizado crescente acerca do assunto. Ademais, ao terminar o game, o participante recebe um certificado, para garantir pontos de acreditação na CNA (Comissão Nacional de Acreditação). 0 jogo está disponível gratuitamente em dispositivos iOs e Android, mas não possui recursos para deficientes visuais (INSUONLINE, 2017).

\subsubsection{Anatogames}

É um excelente método de treinamento para conhecer a anatomia humana, sendo indicado para todos os estudantes de medicina, principalmente os iniciantes do curso. Foi criado em 2009 pelo Laboratório de Anatomia de Sorocaba e, desde então, sua página obteve mais de dois milhões de visualizações. Consiste em nomear corretamente as estruturas indicadas nas peças previamente sinalizadas, 
o que torna essa ferramenta altamente intuitiva e também interativa, uma vez que os erros cometidos pelos usuários são apontados e corrigidos. A plataforma do jogo está vinculada ao Google Tradutor e, portanto, possui tradução para os mesmos idiomas que ele disponibiliza. Além disso, o jogo realiza a classificação por pontos, apresentada através de ranking, o que amplifica a competitividade. Não está disponível para iOS ou Android e não possui recursos para deficientes visuais (ANATOGAMES, 2017).

Na educação médica, os jogos digitais se apresentam em diferentes formatos, como games de simulações, cooperativos e outros que utilizam realidade alternativa. A tecnologia empregada tem o potencial de fornecer experiências virtuais interativas, permitindo criar e avaliar resultados positivos e negativos sobre a proposta do jogo. Observou-se que o uso de games durante o processo de aprendizagem aumenta o nível de concentração dos envolvidos e gera competitividade para consolidar as informações propostas (PETTIT, 2015).

\section{CONSIDERAÇÕES FINAIS}

Constatou-se que as ferramentas interativas abordadas possuem grande potencial de difusão entre acadêmicos do curso de medicina, tendo em vista a facilidade de uso e a acessibilidade. Uma vez que cada recurso possui características, temáticas e abordagens próprias, o aluno pode escolher a mais adequada ao seu atual grau de conhecimento médico e as suas necessidades.

Assim, torna-se interessante, por parte dos docentes, o incentivo à adoção desses tipos de ferramentas para estudo suplementar e autônomo dos alunos, visto que podem contribuir para a consolidação do conhecimento, atuando no processo de aprendizagem mecânica, a partir de termos técnicos e conceitos médicos, e auxiliando no desenvolvimento do raciocínio clínico.

No entanto, vale ressaltar que ainda são escassas as ferramentas de ensino em saúde com opções de utilização por pessoas com deficiência visual, uma vez que apenas uma das nove ferramentas selecionadas apresenta, com limitações, o recurso de áudio para o desenvolvimento de seus recursos didáticos. 
Um estudo exploratório de ferramentas interativas como recurso didático na formação do estudante de medicina

\section{REFERÊNCIAS}

AGgARWAL, R.; MYTTON, T.; DERBREW, M. et al. Training and simulation for patient safety. Qual Saf Health Care, v. 19, n. 2, p. 34-43, 2010.

ALVES, F.P.; MACIEL, C. A gamificação na educação: um panorama do fenômeno em ambientes virtuais de aprendizagem. 2014.

ANATOGAMES. Disponível em: <http://anatogames.blogspot.com/>. Acesso em: 22 ago. 2017.

ASSOCIATION OF AMERICAN MEDICAL COLLEGES. Effective Use of Educational Technology in Medical Education: Summary Report of the 2006 AAMC Colloquium on Educational Technology. Washington, DC: AAMC, p. 7, 2007.

AUSUBEL, D.P. LEARNING THEORY AND CLASSROOM PRACTICE. Ontario Institute for Studies in Education Bulletin, 1967.

BARROSO, C.R.D et al. Análise do uso de simuladores de pacientes virtuais por estudantes de Medicina. Cadernos UniFOA, Volta Redonda, n. 36, p. 91-100, 2018.

BIGDELI, S.; KAUFMAN, D. Digital games in medical education: Key terms, concepts, and definitions. Medical journal of the Islamic Republic of Iran, v. 31, p. 52, 2017.

BIODIGITALHUMAN. Disponível em:<https://www.biodigital.com/>. Acesso em: 2 ago. 2017.

BORGES, T.S.; ALENCAR, G. Metodologias ativas na promoção da formação crítica do estudante: o uso das metodologias ativas como recurso didático na formação crítica do estudante do ensino superior. Cairu em Revista, v. 3, n. 4, p. 119-43, 2014.

DOS SANTOS NUNES, F.L. et al. Realidade Virtual para saúde no Brasil: conceitos, desafios e oportunidades. Rev. Bras. Eng. Biom, v. 27, n. 4, p. 243-58, 2011.

DOS SANTOS, A.D.; DOS SANTOS MACHADO, L. Realidade virtual aplicada ao ensino de medicina: taxonomia, desafios e resultados. 2005.

DOURADO, A.S.S.; GIANNELLA, T.R. Ensino baseado em simulação na formação continuada de médicos: análise das percepções de alunos e professores de um Hospital do Rio de Janeiro. Rev Bras Educ Méd., v. 38, n. 4 , p. $460-469,2014$.

ELECTRONIC VIRTUAL PATIENTS (eVIP). Virtual Patients. Disponível em: <http://virtualpatients.eu/>. Acesso em: 10 set. 2017.

FLATO, U.A.P.; GUIMARÃES, H.P. Educação baseada em simulação em medicina de urgência e emergência: a arte imita a vida. Rev Soc Bras Clin Méd, v. 9, n. 5, p. 360-4, 2011.

GABA, D.M. Do as we say, not as you do: using simulation to investigate clinical behavior in action. Simul Healthc, v. 4, n. 2, p. 67-9, 2009.

INNERBODY. Disponível em:<http://www.innerbody.com/>. Acesso em: 17 ago. 2017. 
INSUONLINE. Disponível em: <https://oge.mobi/insuonline/>. Acesso em: 10 jul 2017.

INTERACTIVE MEDICAL CASES. The New England Journal of Medicine. Disponível em: <http://www. nejm.org/multimedia/interactive-medical-case>. Acesso em: 4 set. 2017.

KAPP, K.M. The gamification of learning and instruction: game-based methods and strategies for training and education. John Wiley \& Sons, 2012.

KHAN, K.; PATTISON, T.; SHERWOOD, M. Simulation in medical education. Med Teach, v. 33, n. 1, p. 1-3, 2011.

MONTEIRO, B. S. et al. AnatomI 3D: Um atlas digital baseado em realidade virtual para ensino de medicina. 2006.

MOORE, M. G. Teoria da Distância Transacional. Revista Brasileira de Educação a Distância, p. 2, 2002.

PERES, C.M.; SUZUKI, K.M.F; DE AZEVEDO-MARQUES, P.M. Recursos tecnológicos de apoio ao ensino na saúde. Medicina (Ribeirao Preto. Online), v. 48, n. 3, p. 224-232, 2015.

PETTIT, R. K.; MCCOY, L., KINNEY, M. et al. Student perceptions of gamified audience response system interactions in large group lectures and via lecture capture technology. BMC medical education, v. 15, n. 1, p. 92, 2015.

RADIOPAEDIA. Disponível em:<https://radiopaedia.org/>. Acesso em: 26 jul. 2017.

SABBATINI, R.M.E. O ensino da informática aplicada à Medicina. Roteiros e bibliografia básica. Rev Informédica, v. 2, n. 8, p. 5-12, 1994.

SOUZA, C.S.; IGLESIAS, A.G.; PAZIN-FILHO, A. Estratégias inovadoras para métodos de ensino tradicionais: aspectos gerais. Medicina (Ribeirão Preto), p. 284-92, 2014.

TORI, R. Educação sem distância: as tecnologias interativas na redução de distâncias em ensino e aprendizagem. São Paulo: Editora SENAC e Escola do Futuro da USP, p. 20, 2010.

TOUCH SURGERY. Disponível em: <https://www.touchsurgery.com/>. Acesso em: 4 set. 2017.

VPSIM. University of Pittsburgh. Disponível em: <http://vpsim.pitt.edu/shell/CaseList_Assignments. aspx>. Acesso em: 20 set. 2017. 